\title{
Higgs-Boson Production via Bottom-Quark Fusion
}

\author{
F. Maltoni ${ }^{1}$ Z. Sullivan, ${ }^{2}$ and S. Willenbrock ${ }^{2,3}$ \\ ${ }^{1}$ Centro Studi e Ricerche "Enrico Fermi" \\ via Panisperna 89/A, 00184 Rome, Italy* \\ ${ }^{2}$ Theoretical Physics Department, Fermi National Accelerator Laboratory, \\ Batavia, IL, 60510-0500 \\ ${ }^{3}$ Department of Physics, University of Illinois at Urbana-Champaign, \\ 1110 West Green Street, Urbana, IL 61801
}

(Dated: January 6, 2003)

\begin{abstract}
Higgs bosons with enhanced coupling to bottom quarks are copiously produced at hadron colliders via $b \bar{b} \rightarrow h$, where the initial $b$ quarks reside in the proton sea. We revisit the calculation of the next-to-leading-order cross section for this process and argue that the appropriate factorization scale for the $b$ distribution functions is approximately $m_{h} / 4$, rather than $m_{h}$, as had been previously assumed. This greatly improves the convergence of the perturbation series, and yields a result with mild factorization-scale dependence. We also show that the leading-order calculation of $g g \rightarrow b \bar{b} h$, integrated over the momenta of the final-state particles, is very sensitive to the factorization and renormalization scales. For scales of order $m_{h} / 4$ the $g g \rightarrow b \bar{b} h$ cross section is comparable to that of $b \bar{b} \rightarrow h$, in contrast to the order-of-magnitude discrepancy between these two calculations for the scale $m_{h}$. The result we obtain improves the prospects for Higgs-boson discovery at hadron colliders for large values of $\tan \beta$.

PACS numbers: 14.80.Bn, 14.80.CP, 12.38.Bx, 13.85.Lg, 13.87.Ce
\end{abstract}

\footnotetext{
*Mail address: Dipartimento di Fisica, Terza Università di Roma, via della Vasca Navale 84, 00146 Rome, Italy
} 


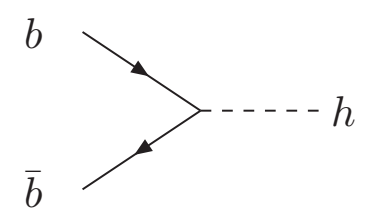

FIG. 1: Leading-order diagram for the production of the Higgs boson via bottom-quark fusion.

\section{INTRODUCTION}

In the standard model, the Higgs boson couples to fermions with strength $m_{f} / v$, where $v=\left(\sqrt{2} G_{F}\right)^{-1 / 2} \approx 246 \mathrm{GeV}$ is the vacuum-expectation value of the Higgs field. The Yukawa coupling of the Higgs boson to bottom quarks $\left(m_{b} \approx 5 \mathrm{GeV}\right)$ is thus very weak, leading to very small cross sections for associated production of the Higgs boson and bottom quarks at the Fermilab Tevatron $(\sqrt{S}=1.96 \mathrm{TeV} p \bar{p})$ [1] and the CERN Large Hadron Collider (LHC, $\sqrt{S}=14 \mathrm{TeV} p p$ ) [2]. However, this Yukawa coupling could be considerably enhanced in extensions of the standard model with more than one Higgs doublet, thereby increasing this production cross section 2]. For example, in a two-Higgs-doublet model, the Yukawa coupling of some or all of the Higgs bosons $\left(h^{0}, H^{0}, A^{0}, H^{ \pm}\right)$to the bottom quark could be enhanced for large values of $\tan \beta=v_{2} / v_{1}$, where $v_{1}$ is the vacuum expectation value of the Higgs doublet that couples to the bottom quark.

The dominant subprocess for the production of a Higgs boson in association with bottom quarks is bottom-quark fusion, $b \bar{b} \rightarrow h$ (Fig. 1) ${ }^{1}$ where the $b$ quarks reside in the proton sea [2, 3, 4]. This is the leading-order (LO) subprocess for the inclusive production of the Higgs boson in association with bottom quarks. Since the bottom-quark sea is generated by gluons splitting into nearly-collinear $b \bar{b}$ pairs, the final state contains two spectator bottom quarks that tend to be at low transverse momentum $\left(p_{T}\right)$.

In contrast, if one requires one bottom quark at high $p_{T}$ from the production process, the leading-order subprocess is $b g \rightarrow b h$ [5, 6, 7]. This process is particularly promising due to the ability to tag the $b$ quark in the final state. The cross section for the production of the Higgs boson accompanied by two high- $p_{T} b$ quarks is obtained at LO from the subprocesses $g g, q \bar{q} \rightarrow b \bar{b} h[8,19,10,11,12,13,14,15] .{ }^{2}$ Although this process has been the most studied,

\footnotetext{
${ }^{1}$ We use $h$ to denote a generic Higgs boson. In a two-Higgs-doublet model, $h$ may denote any of the neutral Higgs bosons $\left(h^{0}, H^{0}, A^{0}\right)$.

${ }^{2} q \bar{q} \rightarrow b \bar{b} h$ is negligible in comparison with $g g \rightarrow b \bar{b} h$.
} 


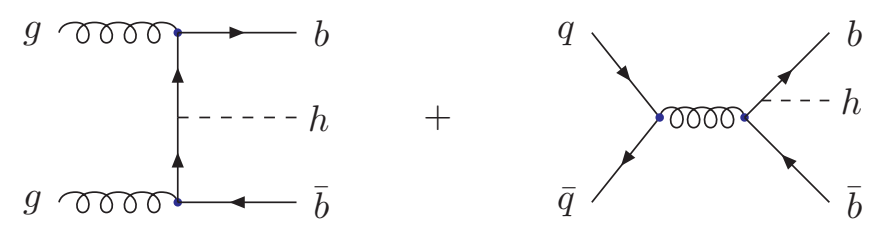

FIG. 2: Representative diagrams for associated production of the Higgs boson and two high- $p_{T}$ bottom quarks: (a) $g g \rightarrow b \bar{b} h$ (8 diagrams); (b) $q \bar{q} \rightarrow b \bar{b} h$ (2 diagrams).

it is likely that $b g \rightarrow b h$ is the more promising, due to its larger cross section. The inclusive cross section, $b \bar{b} \rightarrow h$, which we study in this paper, is useful when the Higgs boson can be identified above backgrounds without the need to detect the accompanying bottom quarks that reside in the final state. This subprocess may be useful to discover a Higgs boson for large $\tan \beta$ in the decay mode $h \rightarrow \tau^{+} \tau^{-}$at the Tevatron and LHC [16, 17] and $h \rightarrow \mu^{+} \mu^{-}$ at the LHC [17, 18, 19]. It has the advantage of having the largest cross section, since it is inclusive of the other two processes.

Higgs-boson production via bottom-quark fusion was calculated at next-to-leading order (NLO) in Refs. [3, 4]. There are two puzzling aspects of the results of that calculation:

- Although the NLO correction is modest, it consists of two independent corrections, of order $1 / \ln \left(m_{h} / m_{b}\right)$ and $\alpha_{S}$, which are both large (and of opposite sign). This suggests that the perturbation series in each expansion parameter individually may not be well behaved.

- The cross section at the Tevatron (both LO and NLO) is an order of magnitude larger than the cross section obtained by calculating $g g \rightarrow b \bar{b} h$ (Fig. 2) and integrating over the momenta of the final-state particles [20]. While $g g \rightarrow b \bar{b} h$ is not a reliable calculation of the total inclusive cross section, since the expansion parameter is $\alpha_{S} \ln \left(m_{h} / m_{b}\right)$ rather than $\alpha_{S}$ [3], the large discrepancy between the two calculations is surprising.

In this paper we solve both of these puzzles. Implicit in both puzzles is the choice of the factorization scale, which had been chosen to be the Higgs-boson mass in Refs. [3, 4]. Although the choice of the factorization scale in a fixed-order calculation is often regarded as arbitrary, we argue that there is a prescription based on physical considerations. Refining the discussion of Ref. [21], we show that the relevant factorization scale is a fraction of the Higgs-boson mass, approximately $m_{h} / 4$. We find that this choice of scale solves both puzzles 
listed above. We thereby present a reliable NLO calculation of the inclusive cross section for the production of the Higgs boson in association with bottom quarks.

In Section [I] we determine the relevant factorization scale for Higgs-boson production in association with bottom quarks. In Section \we present the results of this scale choice. We discuss these results in Section [V] and show that they solve the two puzzles listed above. Section $\nabla$ summarizes the conclusions of our study and suggests further work.

\section{FACTORIZATION SCALE}

The $b$ distribution function, like any parton distribution function, sums (to all orders) collinear logarithms that appear at higher orders. Thus, to determine the relevant factorization scale, we investigate the collinear logarithm that arises at next-to-leading order.

There are two independent NLO corrections to $b \bar{b} \rightarrow h$. The first is from initial gluons, $b g \rightarrow b h$ (Fig. 3), which is a correction of order $1 / \ln \left(m_{h} / m_{b}\right)$, as explained in Ref. [3]. The second is from virtual and real gluon emission (Fig. 4), which is a correction of order $\alpha_{S}$. Since $\alpha_{S}\left(m_{h}\right)$ is proportional to $1 / \ln \left(m_{h} / \Lambda_{Q C D}\right)$, one can regard both of these corrections as being of the form of an inverse logarithm.

The calculations involved are identical to those of Refs. [3, 4]. We keep the effect of the bottom-quark mass exactly in our calculations, without approximations. In order to do this, we may set the bottom-quark mass to zero in all diagrams in which the bottom quark appears as an initial-state parton. ${ }^{3}$ This is called the simplified Aivazis-Collins-Olness-Tung (ACOT) scheme [22, 23, 24]. The only subprocess in which we must keep the bottom-

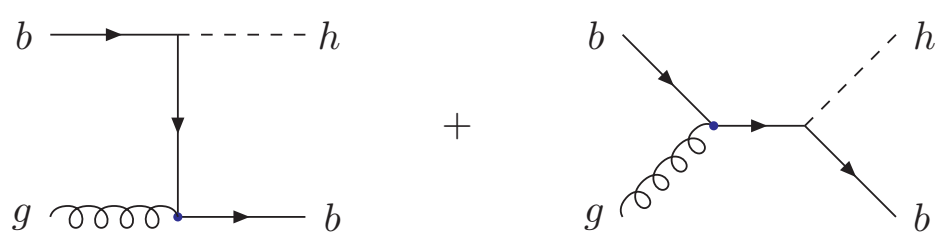

FIG. 3: Diagrams for the next-to-leading-order correction to $b \bar{b} \rightarrow h$ from initial gluons. This correction is of order $1 / \ln \left(m_{h} / m_{b}\right)$.

\footnotetext{
${ }^{3}$ One may maintain a nonzero bottom-quark mass in these diagrams, but it does not increase the accuracy of the calculation.
} 

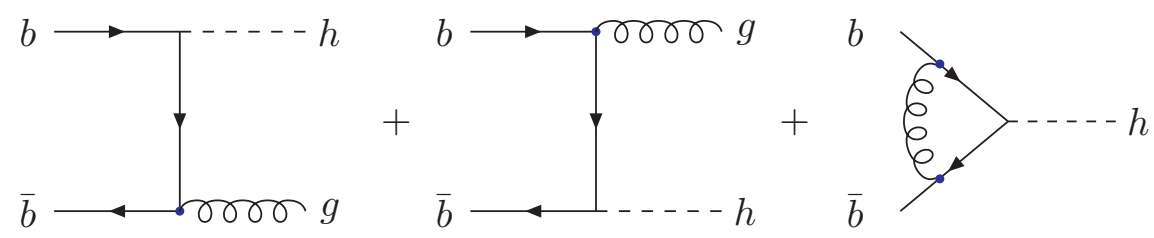

FIG. 4: Diagrams for the next-to-leading-order correction to $b \bar{b} \rightarrow h$ from real and virtual gluon emission. This correction is of order $\alpha_{S}$.

quark mass is $g g \rightarrow b \bar{b} h$ (Fig. (2), which is a next-to-next-to-leading-order correction of order $1 / \ln ^{2}\left(m_{h} / m_{b}\right)$. In practice, for $m_{h} \gg m_{b}$, it is an excellent approximation to neglect the bottom-quark mass in this subprocess; we keep the mass nonzero, nevertheless.

Let us first investigate the $1 / \ln \left(m_{h} / m_{b}\right)$ correction, from initial gluons. The first diagram in Fig. 3 has a collinear divergence due to the $t$-channel quark propagator. The hadronic differential cross section therefore has the behavior $d \sigma / d t \sim 1 / t$ in the collinear region. The integral over $t$ produces the collinear logarithm. Thus, the upper limit of the collinear integration is set by the virtuality, $\sqrt{-t}$, at which the differential cross section begins to deviate substantially from the collinear behavior.

We show in Fig. 5 the hadronic differential cross section times the squared virtuality, $-t d \sigma / d t$, versus the virtuality (scaled to the Higgs-boson mass), $\sqrt{-t} / m_{h}$, for a variety of Higgs-boson masses at the Tevatron and the LHC. In order to compare the cross sections for different Higgs-boson masses at a given collider, we normalize the curves to unity at small virtualities. The curves are nearly identical, demonstrating that the differential cross section scales with the Higgs-boson mass. At small virtualities the curve is flat, indicating the collinear behavior $d \sigma / d t \sim 1 / t$. At larger virtualities the cross section is damped. For fixed $t$, the differential cross section is given by [7]

$\frac{d \sigma}{d t}=-\frac{1}{S} \int_{m_{h}^{2}-t}^{S} d s \int_{\frac{1}{2} \ln (s / S)}^{-\frac{1}{2} \ln (s / S)} d \eta\left[g\left(x_{1}, \mu_{F}\right) b\left(x_{2}, \mu_{F}\right)+\left(x_{1} \leftrightarrow x_{2}\right)\right] \frac{\alpha_{S}\left(\mu_{R}\right)}{24}\left(\frac{y_{b}\left(\mu_{R}\right)}{\sqrt{2}}\right)^{2} \frac{1}{s^{2}} \frac{m_{h}^{4}+u^{2}}{s t}$

which explicitly shows the $1 / t$ behavior for small $t$. For larger values of $-t$, the lower limit on the $s$ integration, $m_{h}^{2}-t$, increases and damps the cross section, since the integrand falls steeply with increasing $s$.

Figure 5 shows that the virtuality at which the behavior of the differential cross section deviates substantially from the collinear behavior is much less than $m_{h}$. Since the curves vary 

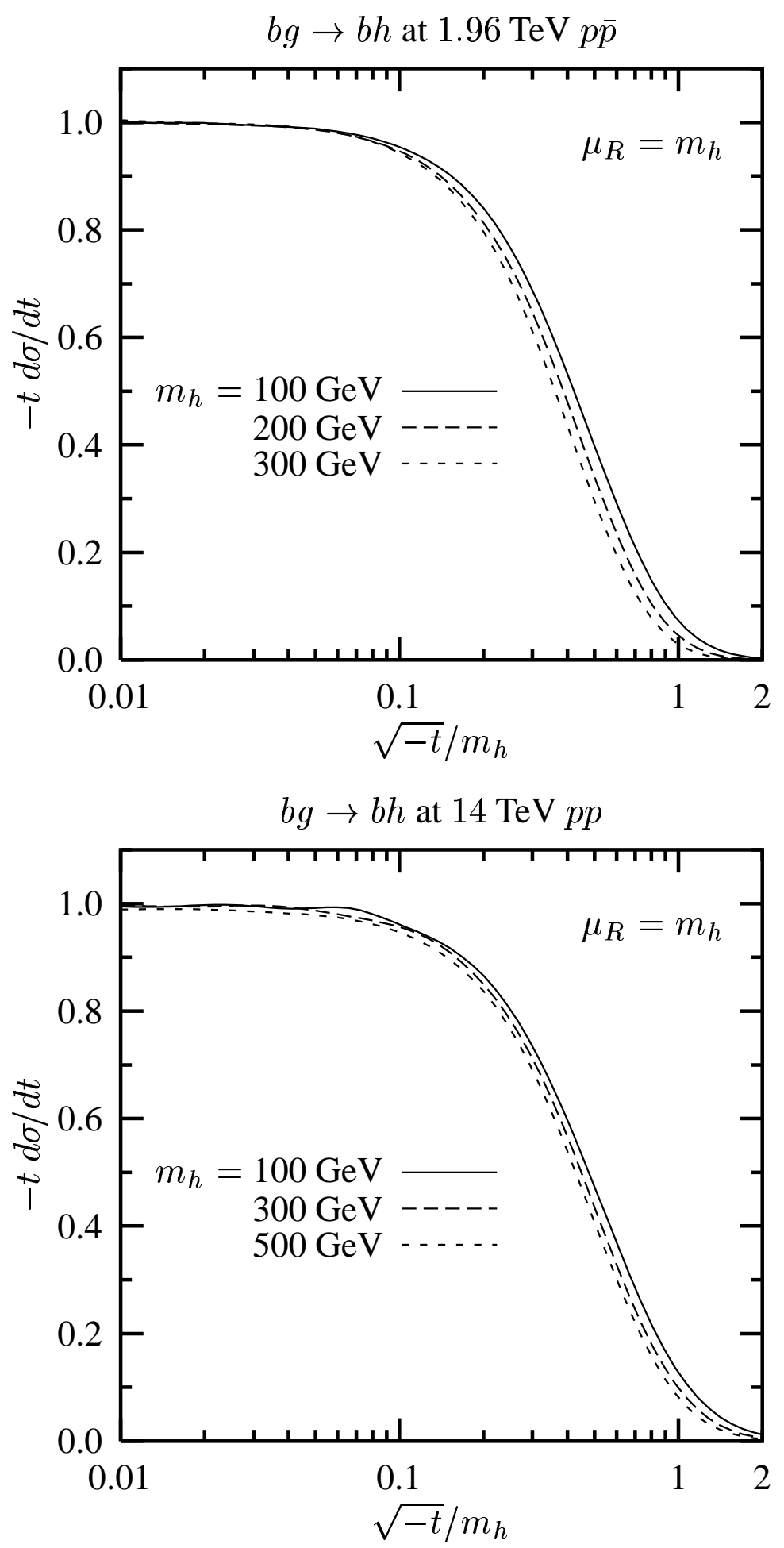

FIG. 5: Hadronic differential cross section times the squared virtuality for the subprocess $b g \rightarrow b h$ vs. the virtuality (scaled to the Higgs-boson mass) at both the Tevatron (upper plot) and the LHC (lower plot). Curves are shown for a variety of Higgs-boson masses, scaled such that they overlap at small virtuality. 
smoothly, there is some ambiguity in defining the virtuality at which the collinear behavior ceases. For the sake of discussion, let us define $\sqrt{-t} \leq m_{h} / 4$ as the collinear region. The collinear region extends to slightly higher virtualities at the LHC than at the Tevatron, but this is small compared with the inherent ambiguity in defining the collinear region.

The collinear logarithm that is generated at NLO is therefore approximately $\ln \left(m_{h} / 4 \mu_{F}\right)$, rather than $\ln \left(m_{h} / \mu_{F}\right)$. Thus the factorization scale for the $1 / \ln \left(m_{h} / m_{b}\right)$ correction should be chosen to be of order $\mu_{F} \approx m_{h} / 4$ in order to sum the collinear logarithm. We will examine the consequences of this scale choice in the next section. In order to account for the ambiguity in defining the collinear region, we will vary the factorization scale between $\mu_{F}=m_{h} / 8$ and $\mu_{F}=m_{h} / 2$.

This derivation of the factorization scale is similar to the argument presented in Ref. 21], where the behavior of the cross section as a function of the transverse momentum of the final-state $b$ quark, $p_{T, b}$, is studied. We prefer to instead use the variable $\sqrt{-t}$, which has the interpretation of the virtuality of the $t$-channel bottom-quark propagator. Since we adopt the simplified ACOT formalism [22, 23, 24], we are able to set the $b$ mass to zero, which makes the discussion of the finite $b$ mass in Ref. [21] moot. Despite these differences, our approach is very similar to that of Ref. [21] and yields similar results.

The results for the $\alpha_{S}$ correction from virtual and real gluon emission (Fig. 4) are similar. We show in Fig. [6 the hadronic differential cross section for real gluon emission times the squared virtuality, $-t d \sigma / d t$, versus the virtuality (scaled to the Higgs-boson mass), $\sqrt{-t} / m_{h}$, for a variety of Higgs-boson masses at the Tevatron and the LHC. A cut of $-u>(10 \mathrm{GeV})^{2}$ is imposed to regulate the infrared singularity associated with soft-gluon emission. The collinear region extends up to a slightly higher virtualities than in the $b g \rightarrow b h$ subprocess, but the difference is small compared with the inherent ambiguity in defining the collinear region. The collinear region again extends to slightly higher virtualities at the LHC than at the Tevatron.

\section{RESULTS}

Following Refs. 3, 4], we fix the renormalization scale of the Yukawa coupling to $\mu_{R}=m_{h}$. It was shown in that paper that the renormalization-scale dependence of the cross section is modest, and is reduced at NLO in comparison with LO. Hence we focus on the factorization- 

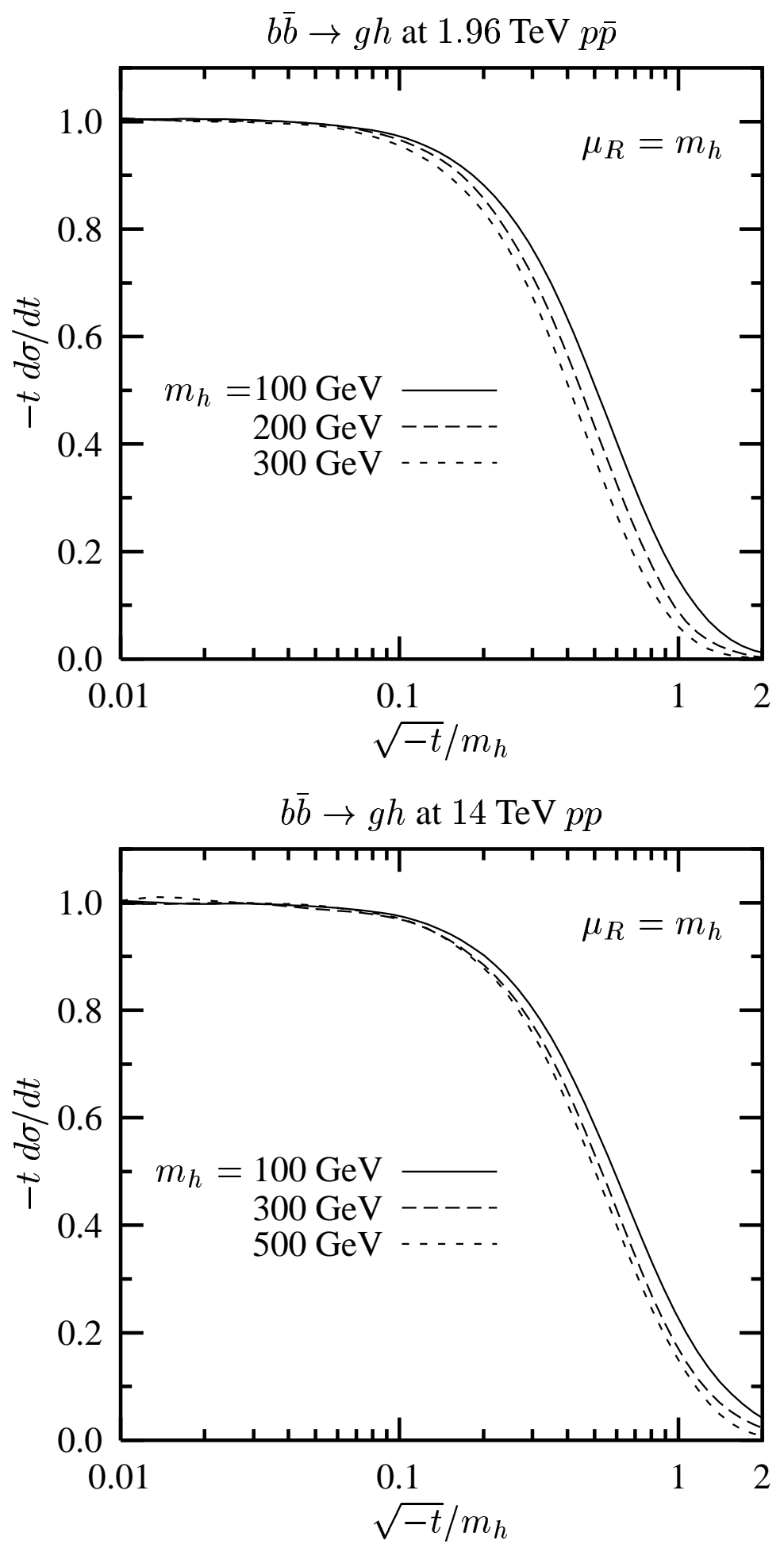

FIG. 6: Same as Fig. 5, but for the subprocess $b \bar{b} \rightarrow g h$. A cut of $-u>(10 \mathrm{GeV})^{2}$ is imposed to regulate the infrared singularity associated with soft-gluon emission. 
scale dependence of the cross section.

We show in Fig. 7 the factorization-scale dependence of the inclusive cross section for Higgs-boson production via bottom-quark fusion for $m_{h}=100 \mathrm{GeV}$ at the Tevatron and the LHC. The four curves on each plot are described below. The results for heavier Higgs bosons are qualitatively similar. While a standard-model Higgs boson of $100 \mathrm{GeV}$ is excluded, the lower bounds on the masses of the neutral Higgs bosons $h^{0}, A^{0}$ of the minimal supersymmetric standard model are about $91 \mathrm{GeV}$ [25]. Even lighter Higgs bosons are possible in a general two-Higgs-doublet model [26]. We set $\tan \beta=1$ throughout.

Let us first focus on the results at the Tevatron, the upper plot in Fig. [7. The factorization scale covers a wide range, including the canonical choice $\mu_{F}=m_{h}$ used in Refs. [3, 4] and $\mu_{F} \approx m_{h} / 4$ advocated in the previous section. The curve labeled "LO" is the LO cross section calculated with LO parton distribution functions (CTEQ6L1 [27]), which has significant factorization-scale dependence. The curve labeled "LO+1/ln" is the partial NLO cross section, including only the $1 / \ln \left(m_{h} / m_{b}\right)$ correction, calculated with NLO parton distribution functions (CTEQ6M). At $\mu_{F}=m_{h}$ this correction is large and negative, approximately $-70 \%$. However, at $\mu_{F} \approx m_{h} / 4$ this correction is small, indicating that this is indeed the relevant factorization scale for this process.

The curve labeled "NLO" in Fig. [7 is the full NLO cross section, including both the $1 / \ln \left(m_{h} / m_{b}\right)$ correction and the $\alpha_{S}$ correction, calculated with NLO parton distribution functions. At $\mu_{F}=m_{h}$, the $\alpha_{S}$ correction is large and positive, nearly canceling the large negative $1 / \ln \left(m_{h} / m_{b}\right)$ correction. However, at $\mu_{F} \approx m_{h} / 4$, the $\alpha_{S}$ correction is modest, yielding a modest NLO correction. This again indicates that this is the relevant factorization scale for this process. The factorization-scale dependence of the NLO cross section is reduced in comparison with that of the LO cross section.

The curve labeled "LO $+1 / \ln +1 / \ln ^{2}$ " in Fig. [7] is the partial NLO cross section [LO plus $1 / \ln \left(m_{h} / m_{b}\right)$ correction] plus the $1 / \ln ^{2}\left(m_{h} / m_{b}\right)$ correction from the diagrams in Fig. $2{ }^{4}$ which is part of the next-to-next-to-leading-order (NNLO) correction. ${ }^{5}$ This NNLO correction is smallest at $\mu_{F} \approx m_{h} / 4$, again indicating that this is the relevant factorization scale for this process. This is significant because while it is always possible to find a factoriza-

\footnotetext{
${ }^{4}$ The correction from $q \bar{q} \rightarrow b \bar{b} h$ is negligible in comparison with that from $g g \rightarrow b \bar{b} h$.

${ }^{5}$ We calculated this curve with NLO parton distribution functions since NNLO parton distribution functions are not yet available.
} 

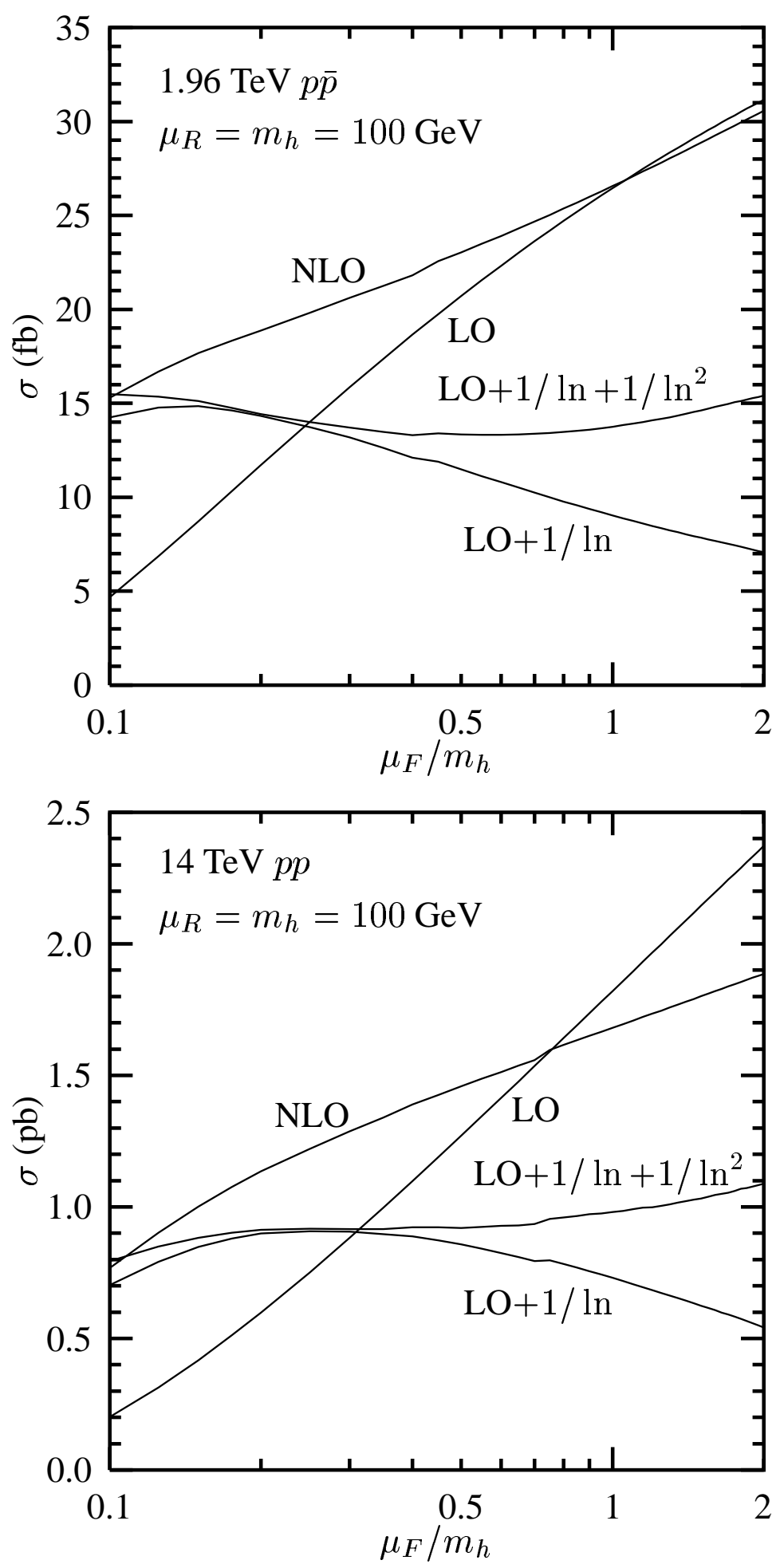

FIG. 7: Cross section for Higgs-boson production via bottom-quark fusion vs. the factorization scale for $m_{h}=100 \mathrm{GeV}$ at the Tevatron (upper plot) and the LHC (lower plot). Curves are shown for leading-order, next-to-leading order, leading-order plus corrections of order $1 / \ln \left(m_{h} / m_{b}\right)$, and leading-order plus corrections of order $1 / \ln \left(m_{h} / m_{b}\right)$ and $1 / \ln ^{2}\left(m_{h} / m_{b}\right)$. 
tion scale such that the NLO correction is small, it is not guaranteed that this same scale will yield a small NNLO correction, unless there is a good motivation for this scale. We anticipate that a full NNLO calculation will further support our argument that the relevant factorization scale for this process is $\mu_{F} \approx m_{h} / 4$.

The results at the LHC, the lower plot in Fig. 7, are qualitatively similar to those at the Tevatron. We argued in the previous section that the relevant factorization scale for this process at the LHC is slightly higher than at the Tevatron. The partial NLO cross section [LO plus $1 / \ln \left(m_{h} / m_{b}\right)$ correction] crosses the LO cross section at a slightly higher factorization scale at the LHC than at the Tevatron, consistent with this argument.

We present in Tables [11] the NLO cross sections for Higgs-boson production via bottomquark fusion at the Tevatron (both $\sqrt{S}=1.8$ and $1.96 \mathrm{TeV}$ ) and the LHC, using $\mu_{F}=m_{h} / 4$. These cross sections differ from those of Refs. [3, 4] in part due to the improved choice of factorization scale, and also in part due to a bug in the CTEQ4M 28] computer code that affected the gluon and $b$ distribution functions used in that paper. Since there is some ambiguity in defining the collinear region, we vary the factorization scale between twice and one-half its central value, and consider this an uncertainty in our calculation. This corresponds to the first uncertainty listed in Tables प프. We also vary the renormalization scale between $m_{h} / 2$ and $2 m_{h}$, and report this as the second uncertainty in Tables 【II. This uncertainty is considerably less than that associated with the factorization scale.

There are two additional sources of uncertainty in our calculation. The uncertainty in the $b$-quark $\overline{\mathrm{MS}}$ mass, $m_{b}\left(m_{b}\right)=4.24 \pm 0.11$ [29], yields an uncertainty in the Yukawa coupling (evaluated at $\mu_{R}=m_{h}$ ). This corresponds to the third uncertainty in Tables 【ய, which is the same for all Higgs-boson masses and machine energies. The fourth uncertainty corresponds to the uncertainty in the parton distribution functions, which we evaluated using the method described in Refs. [27, 30]. The four sources of uncertainty are combined in quadrature and reported as an absolute uncertainty on the NLO cross section. The same exercise is performed for the LO cross section, where we report only the combined uncertainty. 
TABLE I: Leading-order (LO) and next-to-leading-order (NLO) cross sections (pb) for Higgs-boson production via bottom-quark fusion at the Tevatron $(\sqrt{S}=1.8 \mathrm{TeV} p \bar{p})$. The LO cross sections are computed using CTEQ6L1 parton distribution functions [27] and 1-loop evolution of the bottomquark Yukawa coupling, $y_{b}\left(\mu_{R}\right)$. The NLO cross sections are computed using CTEQ6M parton distribution functions and 2-loop evolution of the Yukawa coupling and $\alpha_{S}\left(\mu_{R}\right)$. The four sources of uncertainty in the NLO cross sections are also listed. The factorization scale is $\mu_{F}=m_{h} / 4$, and is varied between $\mu_{F}=m_{h} / 8$ (upper uncertainty) and $\mu_{F}=m_{h} / 2$ (lower uncertainty). The renormalization scale is $\mu_{R}=m_{h}$, and is varied between $\mu_{R}=m_{h} / 2$ (upper uncertainty) and $\mu_{F}=2 m_{h}$ (lower uncertainty). The uncertainty in the Yukawa coupling stems from the uncertainty in the $b$ mass, $m_{b}=4.24 \pm 0.11 \mathrm{GeV}$. The final uncertainty is due to the uncertainty in the parton distribution functions. These four uncertainties are combined in quadrature and reported as an absolute uncertainty in the NLO cross section. The combined uncertainty in the LO cross section is also given.

\begin{tabular}{|c|c|c|c|}
\hline$m_{h}(\mathrm{GeV})$ & $\sigma_{\mathrm{LO}}(\mathrm{pb})$ & $\sigma_{\mathrm{NLO}}(\mathrm{pb})$ & $\delta \sigma_{\mathrm{NLO}}\left(\delta \mu_{F}, \delta \mu_{R}, \delta y_{b}, \mathrm{PDF}\right)(\%)$ \\
\hline 60 & $8.24_{-6.33}^{+7.44} \times 10^{-2}$ & $1.13_{-0.43}^{+0.33} \times 10^{-1}$ & $\begin{array}{ll}-37 & \pm 5.1 \pm 6.4_{-6.4}^{+6.1}\end{array}$ \\
\hline 70 & $4.89_{-3.36}^{+3.63} \times 10^{-2}$ & $6.54_{-1.96}^{+1.67} \times 10^{-2}$ & $\begin{array}{l}-28 \\
+23\end{array} \pm 4.7 \pm 6.4_{-7.4}^{+7.3}$ \\
\hline 80 & $2.99_{-1.86}^{+1.89} \times 10^{-2}$ & $3.92_{-0.98}^{+0.91} \times 10^{-2}$ & 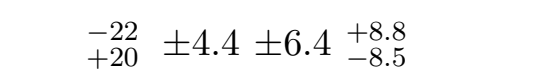 \\
\hline 90 & $1.87_{-1.07}^{+1.05} \times 10^{-2}$ & $2.43_{-0.53}^{+0.53} \times 10^{-2}$ & $\begin{array}{l}-18 \\
+18\end{array} \pm 4.3 \pm 6.4{ }_{-10}^{+10}$ \\
\hline 100 & $1.21_{-0.64}^{+0.61} \times 10^{-2}$ & $1.55_{-0.32}^{+0.33} \times 10^{-2}$ & $\begin{array}{l}-16 \\
+16\end{array} 4.1 \pm 6.4_{-11}^{+12}$ \\
\hline 105 & $9.75_{-5.01}^{+4.69} \times 10^{-3}$ & $1.25_{-0.25}^{+0.27} \times 10^{-2}$ & $\frac{-14}{+15} \pm 4.0 \pm 6.4{ }_{-12}^{+13}$ \\
\hline 110 & $7.92_{-3.95}^{+3.67} \times 10^{-3}$ & $1.022_{-0.20}^{+0.22} \times 10^{-2}$ & ${ }_{+14}^{-13} \pm 3.9 \pm 6.4{ }_{-12}^{+14}$ \\
\hline 115 & $6.48_{-3.13}^{+2.90} \times 10^{-3}$ & $8.28_{-1.61}^{+1.81} \times 10^{-3}$ & ${ }_{+14}^{-12} \pm 3.8 \pm 6.4{ }_{-13}^{+15}$ \\
\hline 120 & $5.32_{-2.51}^{+2.31} \times 10^{-3}$ & $6.79_{-1.31}^{+1.51} \times 10^{-3}$ & ${ }_{+13}^{-12} \pm 3.7 \pm 6.4_{-14}^{+16}$ \\
\hline 125 & $4.38_{-2.01}^{+1.85} \times 10^{-3}$ & $5.60_{-1.09}^{+1.27} \times 10^{-3}$ & ${ }_{+13}^{-11} \pm 3.6 \pm 6.4_{-14}^{+17}$ \\
\hline 130 & $3.63_{-1.63}^{+1.50} \times 10^{-3}$ & $4.63{ }_{-0.91}^{+1.08} \times 10^{-3}$ & 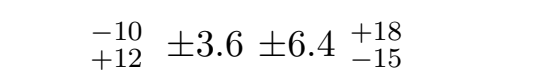 \\
\hline 140 & $2.52_{-1.09}^{+1.01} \times 10^{-3}$ & $3.22_{-0.65}^{+0.80} \times 10^{-3}$ & ${ }_{+12}^{-9.0} \pm 3.4 \pm 6.4_{-17}^{+21}$ \\
\hline 150 & $1.77_{-0.74}^{+0.70} \times 10^{-3}$ & $2.27{ }_{-0.48}^{+0.60} \times 10^{-3}$ & ${ }_{+11}^{-8.0} \pm 3.3 \pm 6.4{ }_{-18}^{+23}$ \\
\hline 160 & $1.26_{-0.51}^{+0.49} \times 10^{-3}$ & $1.62_{-0.36}^{+0.46} \times 10^{-3}$ & ${ }_{+11}^{-7.2} \pm 3.2 \pm 6.4{ }_{-20}^{+25}$ \\
\hline 180 & $6.59_{-2.60}^{+2.65} \times 10^{-4}$ & $8.61_{-2.10}^{+2.79} \times 10^{-4}$ & 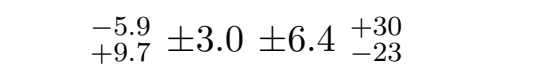 \\
\hline 200 & $3.58_{-1.40}^{+1.53} \times 10^{-4}$ & $4.76_{-1.29}^{+1.76} \times 10^{-4}$ & $\begin{array}{l}-5.0 \\
+8.9\end{array} 2.8 \pm 6.4{ }_{-26}^{+35}$ \\
\hline 250 & $8.78_{-3.63}^{+4.67} \times 10^{-5}$ & $1.24_{-0.43}^{+0.63} \times 10^{-4}$ & $\begin{array}{l}-3.5 \\
+7.7\end{array} 2.6 \pm 6.4_{-34}^{+49}$ \\
\hline 300 & $2.45_{-1.11}^{+1.66} \times 10^{-5}$ & $3.76_{-1.56}^{+2.50} \times 10^{-5}$ & ${ }_{+6.9}^{-2.9} \pm 2.4 \pm 6.4{ }_{-41}^{+66}$ \\
\hline 400 & $2.41_{-1.40}^{+2.55} \times 10^{-6}$ & $4.56_{-2.58}^{+4.81} \times 10^{-6}$ & ${ }_{+5.4}^{-2.7} \pm 2.2 \pm 6.4{ }_{-56}^{+105}$ \\
\hline
\end{tabular}


TABLE II: Same as Table【, but for $\sqrt{S}=1.96 \mathrm{TeV}$.

\begin{tabular}{|c|c|c|c|}
\hline$m_{h}(\mathrm{GeV})$ & $\sigma_{\mathrm{LO}}(\mathrm{pb})$ & $\sigma_{\mathrm{NLO}}(\mathrm{pb})$ & $\delta \sigma_{\mathrm{NLO}}\left(\delta \mu_{F}, \delta \mu_{R}, \delta y_{b}, \mathrm{PDF}\right)(\%)$ \\
\hline 60 & $9.97_{-7.68}^{+9.13} \times 10^{-2}$ & $1.399_{-0.54}^{+0.41} \times 10^{-1}$ & $\begin{array}{ll}-37 & \pm 5.2 \pm 6.4 \\
+28 & +5.9\end{array}$ \\
\hline 70 & $6.00_{-4.13}^{+4.51} \times 10^{-2}$ & $8.13_{-2.47}^{+2.07} \times 10^{-2}$ & $\begin{array}{l}-29 \\
+23\end{array} \pm 4.8 \pm 6.4_{-6.7}^{+6.5}$ \\
\hline 80 & $3.71_{-2.32}^{+2.38} \times 10^{-2}$ & $4.92_{-1.24}^{+1.13} \times 10^{-2}$ & $\begin{array}{l}-23 \\
+20\end{array} \pm 4.5 \pm 6.4_{-7.7}^{+7.7}$ \\
\hline 90 & $2.35_{-1.35}^{+1.33} \times 10^{-2}$ & $3.08_{-0.68}^{+0.66} \times 10^{-2}$ & $\begin{array}{l}-19 \\
+18\end{array} \pm 4.4 \pm 6.4{ }_{-8.8}^{+9.2}$ \\
\hline 100 & $1.53_{-0.82}^{+0.78} \times 10^{-2}$ & $1.98_{-0.40}^{+0.41} \times 10^{-2}$ & $\begin{array}{l}-16 \\
+16\end{array} 4.1 \pm 6.4_{-10}^{+11}$ \\
\hline 105 & $1.24_{-0.64}^{+0.60} \times 10^{-2}$ & $1.61_{-0.32}^{+0.32} \times 10^{-2}$ & $\begin{array}{l}-15 \\
+15\end{array} 4.1 \pm 6.4_{-11}^{+11}$ \\
\hline 110 & $1.02_{-0.51}^{+0.47} \times 10^{-2}$ & $1.31_{-0.25}^{+0.27} \times 10^{-2}$ & $-14 \pm 4.0 \pm 6.4_{-11}^{+12}$ \\
\hline 115 & $8.36_{-4.05}^{+3.76} \times 10^{-3}$ & $1.08_{-0.20}^{+0.22} \times 10^{-2}$ & ${ }_{+14}^{-13} \pm 3.9 \pm 6.4{ }_{-12}^{+13}$ \\
\hline 120 & $6.90_{-3.25}^{+3.00} \times 10^{-3}$ & $8.87_{-1.65}^{+1.84} \times 10^{-3}$ & ${ }_{+13}^{-12} \pm 3.8 \pm 6.4{ }_{-12}^{+14}$ \\
\hline 125 & $5.72_{-2.63}^{+2.42} \times 10^{-3}$ & $7.34_{-1.36}^{+1.55} \times 10^{-3}$ & $\begin{array}{l}-11 \\
+13\end{array} \pm 3.7 \pm 6.4{ }_{-13}^{+15}$ \\
\hline 130 & $4.76_{-2.14}^{+1.96} \times 10^{-3}$ & $6.11_{-1.14}^{+1.32} \times 10^{-3}$ & ${ }_{+12}^{-11} \pm 3.7 \pm 6.4{ }_{-14}^{+16}$ \\
\hline 140 & $3.34_{-1.43}^{+1.32} \times 10^{-3}$ & $4.28_{-0.81}^{+0.97} \times 10^{-3}$ & ${ }_{+12}^{-9.3} \pm 3.5 \pm 6.4{ }_{-15}^{+18}$ \\
\hline 150 & $2.37_{-0.98}^{+0.91} \times 10^{-3}$ & $3.04_{-0.59}^{+0.73} \times 10^{-3}$ & ${ }_{+11}^{-8.3} \pm 3.4 \pm 6.4{ }_{-16}^{+20}$ \\
\hline 160 & $1.70_{-0.69}^{+0.65} \times 10^{-3}$ & $2.19{ }_{-0.45}^{+0.56} \times 10^{-3}$ & ${ }_{+10}^{-7.5} \pm 3.3 \pm 6.4_{-18}^{+22}$ \\
\hline 180 & $9.10_{-3.52}^{+3.47} \times 10^{-4}$ & $1.18_{-0.26}^{+0.34} \times 10^{-3}$ & $\begin{array}{l}-6.2 \\
+9.6\end{array} \pm 3.1 \pm 6.4{ }_{-20}^{+26}$ \\
\hline 200 & $5.05_{-1.92}^{+2.00} \times 10^{-4}$ & $6.66_{-1.64}^{+2.19} \times 10^{-4}$ & $\begin{array}{l}-5.2 \\
+8.9\end{array} 2.9 \pm 6.4{ }_{-23}^{+31}$ \\
\hline 250 & $1.30_{-0.51}^{+0.62} \times 10^{-4}$ & $1.81_{-0.57}^{+0.81} \times 10^{-4}$ & ${ }_{+7.6}^{-3.6} \pm 2.7 \pm 6.4{ }_{-30}^{+43}$ \\
\hline 300 & $3.83_{-1.64}^{+2.30} \times 10^{-5}$ & $5.69_{-2.17}^{+3.31} \times 10^{-5}$ & ${ }_{+6.7}^{-2.9} \pm 2.5 \pm 6.4{ }_{-37}^{+57}$ \\
\hline 400 & $4.21_{-2.26}^{+3.88} \times 10^{-6}$ & $7.48_{-3.87}^{+6.85} \times 10^{-6}$ & ${ }_{+5.2}^{-2.6} \pm 2.2 \pm 6.4_{-51}^{+91}$ \\
\hline 500 & $5.62_{-3.69}^{+7.59} \times 10^{-7}$ & $1.23_{-0.80}^{+1.66} \times 10^{-6}$ & $\begin{array}{l}-2.4 \\
+4.6\end{array} \pm 2.1 \pm 6.4{ }_{-64}^{+134}$ \\
\hline
\end{tabular}

\section{DISCUSSION}

The first puzzle listed in Section \is solved by choosing the factorization scale appropriately. As we showed in the previous section, for $\mu_{F} \approx m_{h} / 4$, the $1 / \ln \left(m_{h} / m_{b}\right)$ correction is small and the $\alpha_{S}$ correction is modest. Thus perturbation theory in each expansion parameter individually is well behaved. Furthermore, we evaluated the $1 / \ln ^{2}\left(m_{h} / m_{b}\right)$ correction as well, and found that it is vanishingly small at the relevant factorization scale, providing 
TABLE III: Same as Table \ but for the LHC $(\sqrt{S}=14 \mathrm{TeV} p p)$.

\begin{tabular}{|c|c|c|c|}
\hline$m_{h}(\mathrm{GeV})$ & $\sigma_{\mathrm{LO}}(\mathrm{pb})$ & $\sigma_{\mathrm{NLO}}(\mathrm{pb})$ & $\delta \sigma_{\mathrm{NLO}}\left(\delta \mu_{F}, \delta \mu_{R}, \delta y_{b}, \mathrm{PDF}\right)(\%)$ \\
\hline 60 & $2.87_{-2.32}^{+3.44} \times 10^{0}$ & $4.69_{-2.35}^{+1.70} \times 10^{0}$ & $\begin{array}{l}-49 \\
+34\end{array} \pm 6.8 \pm 6.4_{-7.3}^{+5.9}$ \\
\hline 70 & $2.04_{-1.51}^{+2.05} \times 10^{0}$ & $3.23_{-1.35}^{+0.99} \times 10^{0}$ & ${ }_{-29}^{-40} \pm 6.3 \pm 6.4{ }_{-7.0}^{+5.5}$ \\
\hline 80 & $1.48_{-1.01}^{+1.29} \times 10^{0}$ & $2.29_{-0.82}^{+0.61} \times 10^{0}$ & $\frac{-34}{+25} \pm 5.8 \pm 6.4{ }_{-6.7}^{+5.2}$ \\
\hline 90 & $1.099_{-0.70}^{+0.85} \times 10^{0}$ & $1.65_{-0.52}^{+0.39} \times 10^{0}$ & $\begin{array}{l}-30 \\
+22\end{array} \pm 5.6 \pm 6.4_{-6.4}^{+5.0}$ \\
\hline 100 & $8.20_{-4.91}^{+5.79} \times 10^{-1}$ & $1.22_{-0.34}^{+0.26} \times 10^{0}$ & $\begin{array}{l}-26 \\
+19\end{array} \pm 5.4 \pm 6.4{ }_{-6.2}^{+4.7}$ \\
\hline 105 & $7.15_{-4.16}^{+4.83} \times 10^{-1}$ & $1.06_{-0.28}^{+0.22} \times 10^{0}$ & $\begin{array}{l}-25 \\
+18\end{array} \pm 5.3 \pm 6.4_{-6.1}^{+4.6}$ \\
\hline 110 & $6.26_{-3.54}^{+4.06} \times 10^{-1}$ & $9.19_{-2.34}^{+1.83} \times 10^{-1}$ & ${ }_{+18}^{-23} \pm 5.2 \pm 6.4_{-6.0}^{+4.6}$ \\
\hline 115 & $5.50_{-3.03}^{+3.44} \times 10^{-1}$ & $8.04_{-1.96}^{+1.55} \times 10^{-1}$ & $\frac{-22}{+17} \pm 5.1 \pm 6.4{ }_{-5.9}^{+4.5}$ \\
\hline 120 & $4.85_{-2.61}^{+2.92} \times 10^{-1}$ & $7.05_{-1.64}^{+1.31} \times 10^{-1}$ & $\begin{array}{ll}-21 & \pm 5.0 \pm 6.4_{-5.8}^{+4.4}\end{array}$ \\
\hline 125 & $4.29_{-2.25}^{+2.50} \times 10^{-1}$ & $6.21_{-1.39}^{+1.12} \times 10^{-1}$ & ${ }_{+16}^{-20} \pm 4.9 \pm 6.4{ }_{-5.7}^{+4.3}$ \\
\hline 130 & $3.81_{-1.96}^{+2.15} \times 10^{-1}$ & $5.48_{-1.18}^{+0.96} \times 10^{-1}$ & $\frac{-19}{+15} \pm 4.9 \pm 6.4_{-5.6}^{+4.3}$ \\
\hline 140 & $3.03_{-1.49}^{+1.61} \times 10^{-1}$ & $4.32{ }_{-0.87}^{+0.71} \times 10^{-1}$ & ${ }_{+14}^{-18} \pm 4.7 \pm 6.4_{-5.5}^{+4.1}$ \\
\hline 150 & $2.44_{-1.15}^{+1.22} \times 10^{-1}$ & $3.45_{-0.65}^{+0.54} \times 10^{-1}$ & $\begin{array}{l}-16 \\
+13\end{array} \pm 4.6 \pm 6.4{ }_{-5.3}^{+4.0}$ \\
\hline 160 & $1.98_{-0.90}^{+0.94} \times 10^{-1}$ & $2.78_{-0.49}^{+0.42} \times 10^{-1}$ & ${ }_{+12}^{-15} \pm 4.5 \pm 6.4_{-5.2}^{+3.9}$ \\
\hline 180 & $1.34_{-0.57}^{+0.58} \times 10^{-1}$ & $1.86_{-0.30}^{+0.26} \times 10^{-1}$ & $\begin{array}{l}-13 \\
+11\end{array} \pm 4.2 \pm 6.4_{-4.9}^{+3.8}$ \\
\hline 200 & $9.31_{-3.73}^{+3.77} \times 10^{-2}$ & $1.29{ }_{-0.19}^{+0.17} \times 10^{-1}$ & $\frac{-12}{+10} \pm 4.1 \pm 6.4_{-4.7}^{+3.7}$ \\
\hline 250 & $4.19_{-1.48}^{+1.45} \times 10^{-2}$ & $5.69{ }_{-0.70}^{+0.66} \times 10^{-2}$ & $\begin{array}{l}-8.9 \\
+8.3\end{array} \pm 3.8 \pm 6.4{ }_{-4.4}^{+3.7}$ \\
\hline 300 & $2.11_{-0.67}^{+0.64} \times 10^{-2}$ & $2.83_{-0.31}^{+0.31} \times 10^{-2}$ & $-7.1 \pm \pm 3.6 \pm 6.4_{-4.3}^{+3.9}$ \\
\hline 400 & $6.66_{-1.81}^{+1.69} \times 10^{-3}$ & $8.81_{-0.88}^{+0.90} \times 10^{-3}$ & ${ }_{+5.6}^{-4.9} \pm 3.3 \pm 6.4_{-5.2}^{+4.9}$ \\
\hline 500 & $2.56_{-0.62}^{+0.58} \times 10^{-3}$ & $3.37_{-0.34}^{+0.36} \times 10^{-3}$ & $-3.6 \pm 3.1 \pm 6.4_{-6.5}^{+6.5}$ \\
\hline 600 & $1.13_{-0.25}^{+0.24} \times 10^{-3}$ & $1.47_{-0.16}^{+0.17} \times 10^{-3}$ & $-2.8 \pm 2.9 \pm 6.4{ }_{-8.0}^{+8.6}$ \\
\hline 700 & $5.42_{-1.16}^{+1.11} \times 10^{-4}$ & $7.12_{-0.85}^{+0.95} \times 10^{-4}$ & ${ }_{+3.8}^{-2.3} \pm 2.8 \pm 6.4{ }_{-10}^{+11}$ \\
\hline 800 & $2.80_{-0.59}^{+0.59} \times 10^{-4}$ & $3.699_{-0.49}^{+0.57} \times 10^{-4}$ & ${ }_{-1.5}^{-1.9} \pm 2.7 \pm 6.4{ }_{-11}^{+13}$ \\
\hline 900 & $1.53_{-0.32}^{+0.34} \times 10^{-4}$ & $2.03_{-0.30}^{+0.36} \times 10^{-4}$ & ${ }_{+3.1}^{-1.5} \pm 2.6 \pm 6.4_{-13}^{+16}$ \\
\hline 1000 & $8.73_{-1.86}^{+2.05} \times 10^{-5}$ & $1.17_{-0.19}^{+0.23} \times 10^{-4}$ & $\begin{array}{l}-1.2 \\
+2.8\end{array} \pm 2.5 \pm 6.4_{-15}^{+19}$ \\
\hline
\end{tabular}


further evidence that the perturbative series in $1 / \ln \left(m_{h} / m_{b}\right)$ is well behaved. This series terminates at this order, while the series in $\alpha_{S}$ extends to all orders [3, 31].

The second puzzle is also solved by a consideration of the choice of scales, both factorization and renormalization. In Refs. 3, 4, 20], all scales were chosen to be the Higgs-boson mass, $\mu_{F}=\mu_{R}=m_{h}$. The NLO cross section at the Tevatron is nearly a factor of ten greater than that obtained by calculating $g g \rightarrow b \bar{b} h$ and integrating over the momenta of the final-state particles. However, this factor is much less at lower scales, mostly because the cross section for $g g \rightarrow b \bar{b} h$ is very scale dependent, and increases significantly at lower scales. In contrast, the NLO cross section for $b \bar{b} \rightarrow h$ has mild scale dependence, and decreases by only about $25 \%$ for $\mu_{F} \approx m_{h} / 4$.

We have established that the relevant factorization scale for $b \bar{b} \rightarrow h$ is $\mu_{F} \approx m_{h} / 4$. It is likely that the relevant factorization scale for $g g \rightarrow b \bar{b} h$ is also much less than $m_{h}$, as well as the renormalization scale of $\alpha_{S}$. We have made no attempt to establish the relevant renormalization scale for the Yukawa coupling in $b \bar{b} \rightarrow h$, and we have found that our NLO calculation is insensitive to this scale. However, the size of the $\alpha_{S}$ correction is less for smaller renormalization scales, which suggests that the relevant renormalization scale may be less than $m_{h}$. Let us adopt this ansatz, although we do not have a rigorous justification for it, in contrast to our derivation of the factorization scale.

As a specific example, we evaluate the cross section for $g g \rightarrow b \bar{b} h$ with $\mu_{F}=\mu_{R}=m_{h}$ and $\mu_{F}=\mu_{R}=m_{h} / 4$ for $m_{h}=100 \mathrm{GeV}$ at the Tevatron, and compare with our NLO calculation of $b \bar{b} \rightarrow h$. The results are listed in Table IV The order of magnitude difference between our NLO calculation and $g g \rightarrow b \bar{b} h$ when the scale is $m_{h}$ is reduced to about a factor of two for scales close to $m_{h} / 4$. A factor of two can easily be accounted for by the fact that our calculation sums collinear logarithms to all orders, while $g g \rightarrow b \bar{b} h$ produces only the LO collinear logarithm. This solves the second puzzle listed in the introduction.

It is the desire to sum collinear logarithms that leads one to use $b \bar{b} \rightarrow h$ as the LO subprocess for the inclusive production of the Higgs boson. The corrections are of order $1 / \ln \left(m_{h} / m_{b}\right)$ and $\alpha_{S}$, and we have seen that they are modest for the appropriate choice of factorization scale. If one were to use $g g \rightarrow b \bar{b} h$ as the LO subprocess, the expansion parameter would be $\alpha_{S} \ln \left(m_{h} / m_{b}\right)$, and perturbation theory would be poorly behaved. Our calculation gives the most accurate and reliable cross section for the inclusive production of the Higgs boson because it sums these collinear logarithms to all orders. 
TABLE IV: Cross sections (fb) for $b \bar{b} \rightarrow h$ at NLO and $g g \rightarrow b \bar{b} h$ at LO for $m_{h}=100 \mathrm{GeV}$ at the Tevatron, for two choices of the common factorization and renormalization scales. The final column gives the ratio of the cross sections. The ratio is nearly an order of magnitude for $\mu_{F}=\mu_{R}=m_{h}$, but is only about a factor of two for $\mu_{F}=\mu_{R}=m_{h} / 4$.

\begin{tabular}{ccc|c}
\hline \hline Scales & $\sigma(b \bar{b} \rightarrow h)$ & $\sigma(g g \rightarrow b \bar{b} h)$ & $\sigma(b \bar{b} \rightarrow h) / \sigma(g g \rightarrow b \bar{b} h)$ \\
\hline$\mu_{F}=\mu_{R}=m_{h}$ & $26.6 \mathrm{fb}$ & $3.1 \mathrm{fb}$ & 8.5 \\
$\mu_{F}=\mu_{R}=m_{h} / 4$ & $20.8 \mathrm{fb}$ & $9.2 \mathrm{fb}$ & 2.3 \\
\hline \hline
\end{tabular}

It is suggested in Refs. [32, 33] that the calculation of $b \bar{b} \rightarrow h$ may overestimate the inclusive cross section, due to crude approximations inherent in the kinematics, which give rise to large bottom-quark mass and phase-space effects. However, the ACOT formalism [22, 23, 24] makes no approximations in either the kinematics or the $b$ mass; we maintained the effect of the $b$ mass exactly. We find no evidence for any inconsistency in the ACOT formalism. Rather, we find that the LO calculation of $g g \rightarrow b \bar{b} h$, integrated over the momenta of the final-state particles, underestimates the inclusive cross section when the factorization and renormalization scales are chosen to be $m_{h}$.

\section{CONCLUSIONS}

We have revisited the next-to-leading-order (NLO) calculation of Higgs-boson production via bottom-quark fusion and solved the two puzzles associated with that calculation [3, 4]. We showed that the appropriate factorization scale for this process is $\mu_{F} \approx m_{h} / 4$, rather than $m_{h}$, as had been previously assumed. This greatly improves the convergence of the perturbation series, which was mediocre for $\mu_{F}=m_{h}$. The resulting cross section has mild factorization-scale dependence, and small renormalization-scale dependence. It is the most reliable calculation of the inclusive cross section for Higgs-boson production in association with $b$ quarks.

To support our arguments, we calculated one of the next-to-next-to-leading (NNLO) corrections (associated with the diagrams in Fig. 2), and showed that it is vanishingly small for $\mu_{F} \approx m_{h} / 4$. The ingredients exist to calculate the full NNLO cross section, using the 
results from Refs. 7, 34]. This should yield a cross section with small factorization- and renormalization-scale dependence. It will also provide an additional check of our choice of factorization scale.

The other puzzle we solved also involves the choice of scales, both factorization and renormalization. The inclusive cross section for Higgs-boson production in association with bottom quarks may be approximated by $g g \rightarrow b \bar{b} h$, integrated over the momenta of the finalstate particles. This yields a result an order of magnitude less than the NLO calculation of $b \bar{b} \rightarrow h$. However, this result is very scale dependent, since it is based on a leading-order calculation. Choosing scales of order $m_{h} / 4$ rather than $m_{h}$, we find that the cross section is comparable to that of $b \bar{b} \rightarrow h$ (see Table [V]). The NLO calculation of $g g \rightarrow b \bar{b} h$ might support these observations. However, that calculation is not as accurate as one based on $b \bar{b} \rightarrow h$ for $m_{h} \gg m_{b}$, since the latter sums collinear logarithms to all orders in perturbation theory.

Let us review the existing calculations of Higgs-boson production in association with bottom quarks. The relevant calculation depends upon the final state that is desired. For the inclusive cross section, the relevant leading-order (LO) subprocess is $b \bar{b} \rightarrow h$ (Fig. (1). The NLO cross section was calculated in Refs. [3, 4] and updated in this paper. The cross section for the production of the Higgs boson accompanied by one high-transverse-momentum $\left(p_{T}\right)$ bottom quark is obtained at LO from the subprocess $b g \rightarrow b h$ (Fig. 31), which is calculated at NLO in Ref. 7]. This process is particularly promising due to the ability to tag the $b$ quark in the final state. Finally, the cross section for the production of the Higgs boson accompanied by two high- $p_{T} b$ quarks is obtained at LO from the subprocesses $g g, q \bar{q} \rightarrow b \bar{b} h$ (Fig. 2). This process has been calculated only at LO thus far, but the ingredients exist to provide the NLO cross section [35, 36, 37, 38, 39]. Although this process has been the most studied, it is likely that $b g \rightarrow b h$ is the more promising, due to its larger cross section. The inclusive cross section, $b \bar{b} \rightarrow h$, obtained in this paper, is useful when the Higgs boson can be identified above backgrounds without the need to tag $b$ quarks, such as $h \rightarrow \tau^{+} \tau^{-}, \mu^{+} \mu^{-}$. It has the advantage of having the largest cross section, since it is inclusive of the other two processes. 


\section{Acknowledgments}

We are grateful for conversations and correspondence with E. Braaten, A. Connolly, D. Froidevaux, T. Junk, P. Lepage, P. Mackenzie, and E. Richter-Was. F. M. warmly thanks the Department of Physics of the "Terza Università di Roma" for the kind hospitality and support. This work was supported in part by the U. S. Department of Energy under contracts Nos. DE-FG02-91ER40677 and DE-AC02-76CH03000.

[1] A. Stange, W. J. Marciano and S. Willenbrock, Phys. Rev. D 49, 1354 (1994) arXiv:hep-ph/9309294.

[2] D. A. Dicus and S. Willenbrock, Phys. Rev. D 39, 751 (1989).

[3] D. Dicus, T. Stelzer, Z. Sullivan and S. Willenbrock, Phys. Rev. D 59, 094016 (1999) arXiv:hep-ph/9811492.

[4] C. Balazs, H. J. He and C. P. Yuan, Phys. Rev. D 60, 114001 (1999) arXiv:hep-ph/9812263.

[5] D. Choudhury, A. Datta and S. Raychaudhuri, arXiv:hep-ph/9809552.

[6] C. S. Huang and S. H. Zhu, Phys. Rev. D 60, 075012 (1999) arXiv:hep-ph/9812201.

[7] J. Campbell, R. K. Ellis, F. Maltoni and S. Willenbrock, arXiv:hep-ph/0204093.

[8] J. Dai, J. F. Gunion and R. Vega, Phys. Lett. B 345, 29 (1995) arXiv:hep-ph/9403362.

[9] J. Dai, J. F. Gunion and R. Vega, Phys. Lett. B 387, 801 (1996) arXiv:hep-ph/9607379.

[10] E. Richter-Was and D. Froidevaux, Z. Phys. C 76, 665 (1997) arXiv:hep-ph/9708455.

[11] M. Drees, M. Guchait and P. Roy, Phys. Rev. Lett. 80, 2047 (1998) [Erratum-ibid. 81, 2394 (1998)] arXiv:hep-ph/9801229.

[12] J. L. Diaz-Cruz, H. J. He, T. Tait and C. P. Yuan, Phys. Rev. Lett. 80, 4641 (1998) arXiv:hep-ph/9802294.

[13] C. Balazs, J. L. Diaz-Cruz, H. J. He, T. Tait and C. P. Yuan, Phys. Rev. D 59, 055016 (1999) arXiv:hep-ph/9807349.

[14] M. Carena, S. Mrenna and C. E. Wagner, Phys. Rev. D 60, 075010 (1999) arXiv:hep-ph/9808312.

[15] S. Dawson, D. Dicus and C. Kao, Phys. Lett. B 545, 132 (2002) arXiv:hep-ph/0208063.

[16] Z. Kunszt and F. Zwirner, Nucl. Phys. B 385, 3 (1992) arXiv:hep-ph/9203223.

[17] ATLAS Collaboration, Technical Design Report, CERN-LHCC-99-15. 
[18] C. Kao and N. Stepanov, Phys. Rev. D 52, 5025 (1995) arXiv:hep-ph/9503415.

[19] V. D. Barger and C. Kao, Phys. Lett. B 424, 69 (1998) arXiv:hep-ph/9711328.

[20] M. Carena et al., Report of the Tevatron Higgs Working Group, arXiv:hep-ph/0010338.

[21] T. Plehn, Phys. Rev. D 67, 014018 (2003) arXiv:hep-ph/0206121.

[22] M. A. Aivazis, J. C. Collins, F. I. Olness and W. K. Tung, Phys. Rev. D 50, 3102 (1994) arXiv:hep-ph/9312319.

[23] J. C. Collins, Phys. Rev. D 58, 094002 (1998) arXiv:hep-ph/9806259.

[24] M. Kramer, F. I. Olness and D. E. Soper, Phys. Rev. D 62, 096007 (2000) arXiv:hep-ph/0003035.

[25] LEP Higgs Working Group Collaboration, arXiv:hep-ex/0107030.

[26] P. Ferrari and D. Zer-Zion [OPAL Collaboration], "Two Higgs Doublet Model Interpretation of Neutral Higgs Boson Searches up to the Highest LEP Energies," OPAL Physics Note PN475 (2001).

[27] J. Pumplin, D. R. Stump, J. Huston, H. L. Lai, P. Nadolsky and W. K. Tung, JHEP 0207, 012 (2002) arXiv:hep-ph/0201195.

[28] H. L. Lai et al., Phys. Rev. D 55, 1280 (1997) arXiv:hep-ph/9606399.

[29] A. X. El-Khadra and M. Luke, arXiv:hep-ph/0208114.

[30] Z. Sullivan, Phys. Rev. D 66, 075011 (2002) arXiv:hep-ph/0207290.

[31] T. Stelzer, Z. Sullivan and S. Willenbrock, Phys. Rev. D 56, 5919 (1997) arXiv:hep-ph/9705398.

[32] D. Rainwater, M. Spira and D. Zeppenfeld, arXiv:hep-ph/0203187.

[33] M. Spira, arXiv:hep-ph/0211145.

[34] K. Melnikov, Phys. Rev. D 53, 5020 (1996) arXiv:hep-ph/9511310.

[35] W. Beenakker, S. Dittmaier, M. Kramer, B. Plumper, M. Spira and P. M. Zerwas, Phys. Rev. Lett. 87, 201805 (2001) arXiv:hep-ph/0107081.

[36] W. Beenakker, S. Dittmaier, M. Kramer, B. Plumper, M. Spira and P. M. Zerwas, arXiv:hep-ph/0211352,

[37] L. Reina and S. Dawson, Phys. Rev. Lett. 87, 201804 (2001) arXiv:hep-ph/0107101.

[38] L. Reina, S. Dawson and D. Wackeroth, Phys. Rev. D 65, 053017 (2002) arXiv:hep-ph/0109066.

[39] S. Dawson, L. H. Orr, L. Reina and D. Wackeroth, arXiv:hep-ph/0211438 